\title{
ACTIVE GALACTIC NUCLEUS OBSCURATION FROM WINDS: FROM DUSTY INFRARED-DRIVEN TO WARM AND X-RAY PHOTOIONIZED
}

\author{
A. DORODNitsyn ${ }^{1,2}$ AND T. KaLlman ${ }^{1}$ \\ ${ }^{1}$ Laboratory for High Energy Astrophysics, NASA Goddard Space Flight Center, Code 662, Greenbelt, MD 20771, USA \\ 2 Department of Astronomy/CRESST, University of Maryland, College Park, MD 20742, USA \\ Received 2012 August 27; accepted 2012 October 14; published 2012 November 27
}

\begin{abstract}
We present calculations of active galactic nucleus winds at $\sim$ parsec scales along with the associated obscuration. We take into account the pressure of infrared radiation on dust grains and the interaction of X-rays from a central black hole with hot and cold plasma. Infrared radiation (IR) is incorporated in radiation-hydrodynamic simulations adopting the flux-limited diffusion approximation. We find that in the range of X-ray luminosities $L=0.05-0.6$ $L_{\text {Edd }}$, the Compton-thick part of the flow (aka torus) has an opening angle of approximately $72^{\circ}-75^{\circ}$ regardless of the luminosity. At $L \gtrsim 0.1$, the outflowing dusty wind provides the obscuration with IR pressure playing a major role. The global flow consists of two phases: the cold flow at inclinations $\theta \gtrsim 70^{\circ}$ and a hot, ionized wind of lower density at lower inclinations. The dynamical pressure of the hot wind is important in shaping the denser IR-supported flow. At luminosities $\leqslant 0.1 L_{\text {Edd }}$ episodes of outflow are followed by extended periods when the wind switches to slow accretion.
\end{abstract}

Key words: acceleration of particles - galaxies: active - hydrodynamics - methods: numerical

Online-only material: color figures

\section{INTRODUCTION}

Active galactic nuclei (AGNs) are among the most fascinating objects in the universe: various energetic processes interplay in a volume of a few cubic parsecs around a supermassive black hole $(\mathrm{BH})$; radiation is likely decisive in connecting the accretion and feedback modes of AGNs. Dusty, radiation-driven flows are very effective in removing large masses of accreting gas, returning it back to the galaxy, and naturally limiting the growth rate of a supermassive $\mathrm{BH}$ by the feedback with the host galaxy. Radiation also provides a nonlinear causal connection between distant parts of an AGN itself, coupling together vastly different physical scales. Despite the pivotal role of radiation in the first-principles modeling of AGNs and the significant progress in theoretical modeling over the past 10 years, proper incorporation of radiation into numerical models still presents a significant computational challenge.

Well-established phenomenology places AGNs into two distinct classes that roughly differ by the presence (type I) or the absence (type II) of the broad emission lines in the optical and UV spectra. According to the AGN unification paradigm (Rowan-Robinson 1977; Antonucci \& Miller 1985; Urry \& Padovani 1995), this dichotomy is an artifact of a pure geometrical origin. In order to be observed as a type II object, an AGN must be viewed at high inclination, in extreme cases close to the equatorial plane, while in the case of a type I AGN the observer's line of sight is at lower inclination, i.e., closer to the symmetry axis. The presence of geometrically thick toroidal obscuration is pivotal to the unification scheme of AGNs.

The discovery of broad permitted emission lines in the polarized spectrum of the nearby Seyfert II galaxy NGC 1068 (Antonucci 1984; Antonucci \& Miller 1985) provides evidence that a type I nucleus is harbored inside the obscuring ring of matter. The observed polarization is likely due to scattering of light emitted by the type I core and then being scattered within an outflow emanating from the same nucleus.
Observations of AGNs and quasars reveal that dust in large quantities can be present within a few parsecs from the $\mathrm{BH}$, and a significant part of X-rays and UV are reprocessed into infrared radiation (IR). For example, in luminous quasars almost half of the bolometric luminosity can be reprocessed into IR. This results in a 1-100 $\mu \mathrm{m}$ "hump" in the quasar spectral energy distribution (Richards et al. 2006). In nearby AGNs, multitemperature dust distributions are observed directly by the Very Large Telescope Interferometer. Such mid-infrared observations of the prototypical Seyfert II galaxy NGC 1068 (Jaffe et al. 2004) and the Circinus galaxy (Tristram et al. 2007) depict a dusty, clumpy plasma exposed to external illumination.

Various models have been proposed to explain the real or the apparent thickness of the torus:

1. A "Warped disk model": these are global warps in a locally geometrically thin accretion disk (i.e., Phinney 1989; Sanders et al. 1989).

2. (i) Models of a quasi-static, rotating torus involving "clouds" (i.e., Krolik \& Begelman 1988; Beckert \& Duschl 2004; Nenkova et al. 2008). Vertical thickness of the torus is supported via a large velocity dispersion of clouds, and a small-scale magnetic field is required to maintain elasticity of clouds during collisions.

(ii) Models that rely on IR pressure on dust to keep the rotating torus vertically thick (Krolik 2007). Most of the IR in this model comes from reprocessing of external X-ray and UV radiation.

3. A "wind torus": a collar of outflowing matter may provide obscuration.

(i) MHD wind: the possibility is that a global magnetic field can be involved in the form of an MHD wind (Konigl \& Kartje 1994; Elitzur \& Shlosman 2006) or directly supporting a quasi-static torus (Lovelace et al. 1998), or in a combination of radiative and magnetic driving (Emmering et al. 1992; Everett 2005; Keating et al. 2012). Note that a sufficiently strong (of the order of the equipartition one) 
poloidal magnetic field can also help to solve the problem of how the angular momentum is removed from accreting matter; however, the origin of such a highly ordered field remains unclear.

(ii) An outflow (or failed wind) driven by IR pressure on dust (Dorodnitsyn et al. 2011, 2012). All these models have their benefits and drawbacks.

Models investigating the properties of a self-gravitating, nonradiative, dissipationless torus through $N$-body simulations have been made by Bannikova et al. (2012).

The accreting dusty gas at $\sim$ parsec distances from the $\mathrm{BH}$ is susceptible to self-gravitating instability; since it is exposed to intense radiation, strong gravitothermal instabilities can develop.

In the outflowing wind, the nonlinear phase of thermal instability is associated with "clumps" (i.e., Elitzur 2008). Presumably such clouds are confined by a small-scale magnetic field. Whether they are secondary to the flow and behave more like "waves," i.e., temporary structures evolving on dynamical or radiation timescales, or real long-lasting "clumps" remains unresolved. All models involving clouds need to explain how to avoid too much dissipation through the cloud-cloud collisions, which would otherwise raise the temperature to a fraction of the virial temperature $T_{\mathrm{vir}, \mathrm{g}}$. In the present studies, we do not consider the effect of the magnetic field (both ordered and chaotic).

It has long been recognized that the geometrical thickness of the obscuration presents a significant problem. In order to explain why the torus is geometrically thick, models have been constructed that rely on gas pressure, magnetic forces, and radiation pressure either alone or in combination with other mechanisms. If one relies on gas pressure to support vertical thickness of the torus, then virial arguments suggest that the temperature of such gas should be of the order of $T_{\mathrm{vir}, \mathrm{g}}=2.6 \times 10^{6} M_{7} / r_{\mathrm{pc}} \mathrm{K}$, where $M_{7}$ is the $\mathrm{BH}$ mass in $10^{7} M_{\odot}$ and $r_{\mathrm{pc}}$ is the distance in parsecs. Such high temperatures are not compatible with the existence of dust.

As mentioned before, the presence of a small-scale magnetic field can provide necessary elasticity to cloud-cloud collisions. However, this argument is not without a flaw. It is natural to assume that turbulence develops. If so, this leads to the magnetic field being of the order of the equipartition field. A result is that the dissipation of magnetosonic waves will raise the electron temperature to be of the order of $T_{\mathrm{vir}, \mathrm{g}}$.

The connection between a toroidal obscuration, UV absorption, and warm absorbers can be self-consistently assessed by global multi-dimensional simulations. The prequisite for such an endeavor is to include various physical effects and couple them to radiation. Additionally, why such calculations are currently beyond reach is that high numerical resolution is needed to resolve the multiphase behavior of the outflowing gas. These are the reasons why different works including present studies assess smaller aspects of the bigger picture.

The connection between a broad-line region and dusty outflow was considered by Czerny \& Hryniewicz (2011). They suggested that the gas of the dusty accretion disk is weakly coupled to the $\mathrm{BH}$ due to radiation pressure on dust, resulting in a dusty wind that gives the low-ionization part of the broad-line region. Bottorff et al. (2000) considered the relation between the observed properties of UV and X-ray absorbers and those predicted from a magnetohydrodynamical self-similar wind model. They conclude that in the case of the well-studied Seyfert 1 galaxy NGC 5548 the intrinsically clumped absorber is biased toward smaller radii in the case of warm absorbers and is likely located at higher distances in the case of UV absorption features.

Other models are based on the possibility that the pressure of the infrared radiation on dust may itself be enough to support the vertical thickness of the torus. The idea of a static infrared support was explored in Krolik (2007), where an approximate analytical model for the torus was constructed showing that there is enough radiation force to support a static vertically thick torus. However, it was argued in Dorodnitsyn et al. (2011, hereafter Paper I) that it is very unlikely that the IR-supported torus would be static. It was shown that the equilibrium between rotational, gravitational, and radiation forces cannot be maintained if the temperature in the torus exceeds that of $T_{\mathrm{vir}, \mathrm{r}} \simeq 312\left(n / 10^{5} M_{7} r_{\mathrm{pc}}^{-1}\right)^{1 / 4}-987\left(n / 10^{7} M_{7} r_{\mathrm{pc}}^{-1}\right)^{1 / 4} \mathrm{~K}$, where $n$ is the number density, necessarily resulting in the torus being an outflow. In the following paper (Dorodnitsyn et al. 2012, hereafter Paper II), we supported this idea with radiation-hydrodynamic simulations of the outflowing accretion disk wind, driven by the radiation pressure on dust. The distribution of IR was calculated using a flux-limited diffusion approximation.

An important simplification was made in Papers I and II: only the infrared-driven part of the wind was considered. The argument was that this portion of the wind is far enough from the $\mathrm{BH}$ so that all the conversion of X-rays to IR already happened outside the computational domain. That allowed us to consider only one group of radiation (the IR). The radiation temperature at the boundary was calculated to match the input energetics of soft X-rays. In other words, we did not treat explicitly the reprocessing of soft X-rays into IR. Correspondingly, we were not able to follow the interaction of the X-ray-heated hot component of the flow with the cold, dusty IR-supported component.

In this paper, we calculate the wind structure and explicitly treat the interaction between X-rays and gas with subsequent conversion of X-rays to IR. This allows us to follow in dynamics the transition from hot, photoionized to cold, dusty and IR-supported flow. To make calculations numerically tractable, we made several major simplifications in treatment of the transfer of X-rays and their interaction with the gas. For example, transfer of X-rays is reduced to simple attenuation with no multiple scatterings taken into account, and we approximately calculate the heating of dust grains by X-rays. The rest of our computational radiation-hydrodynamic framework remains unchanged from Paper II: the transfer of the infrared radiation and its interaction with matter are treated in a flux-limited diffusion approximation. The calculations are time-dependent and 2.5D, i.e., three dimensions in cylindrical coordinates and assuming axial symmetry.

The organization of this paper is as follows. After this introduction, in Section 2 we review basic assumptions of our model, including the description of the radiating fluid of the AGN dusty wind by means of radiation-hydrodynamic equations. Interaction of the cold and hot components of the wind with X-rays is described in Section 3, including corresponding approximations. In Section 4 we describe the numerical setup and initial and boundary conditions (BCs). The results are summarized in Section 5, the obscuring properties of the wind and the mass loss rates are discussed in Sections 7 and 8; the results are summarized in the Discussion; the place of our 
model to a broader perspective of AGN parsec-scale flows and the final comments are given in the Conclusions section.

\section{ASSUMPTIONS AND PHYSICAL MODEL}

The flow in which the continuum radiation plays an important role is described by the system of radiation-hydrodynamic equations. To first order in $v / c$, these can be formulated in the following form (Mihalas \& Mihalas 1984):

$$
\begin{gathered}
D_{t} \rho+\rho \nabla \cdot \mathbf{v}=0 \\
D_{t} \mathbf{v}=-\frac{1}{\rho} \nabla p+\mathbf{g}_{\mathrm{rad}}-\nabla \Phi \\
\rho D_{t}\left(\frac{e}{\rho}\right)=-p \nabla \cdot \mathbf{v}-4 \pi \chi_{\mathrm{P}} B+c \chi_{\mathrm{E}} E+n^{2}(\Gamma-\Lambda), \\
\rho D_{t}\left(\frac{E}{\rho}\right)=-\nabla \cdot \mathbf{F}-\nabla \mathbf{v}: \mathbf{P}+4 \pi \chi_{\mathrm{P}} B-c \chi_{\mathrm{E}} E
\end{gathered}
$$

where quantities related to matter are as follows: $\rho, p$, and $e$ are the material mass density, gas pressure, and gas energy density, respectively, and $\mathbf{v}$ is the velocity; quantities related to radiation are the frequency-integrated moments (for definitions see Appendix A): $E$ is the radiation energy density, $\mathbf{F}$ is the radiation flux, and $\mathbf{P}$ is the radiation pressure tensor; $\chi_{\mathrm{P}}, \chi_{\mathrm{E}}$ are the Planck mean and energy mean absorption opacities (in $\left.\mathrm{cm}^{-1}\right), c$ is the speed of light, $B=\sigma T^{4} / \pi$ is the Planck function, $\sigma=a c / 4$ is the Stefan-Boltzmann constant, and $T$ is the gas temperature; other notation includes the convective derivative, $D_{t}=(\partial / \partial t)+\mathbf{v} \cdot \nabla$, and $\nabla \mathbf{v}: \mathbf{P}$ denotes the contraction $\left(\partial_{j} v_{i}\right) P^{i j}$. Note that all the dependent variables in (1)-(4) are evaluated in the comoving frame. Note that $E=a T_{\mathrm{r}}^{4}$, where $T_{\mathrm{r}}$ is the radiation temperature. Interaction between $\mathrm{X}$-rays and the gas is incorporated in the last term of the gas energy equation (3), where $\Gamma$ and $\Lambda$ are, respectively, heating and cooling rates. The equation of state for the gas is assumed to be polytropic: $p=K \rho \gamma$, where $\gamma=1+1 / n, n$ is the polytrope index, and $p=(\gamma-1) e$. The gas is one-component, one-temperature $T=p \mu / \rho \mathcal{R}$, where $\mu$ is the mean molecular weight per particle, $\mathcal{R}=8.31 \times 10^{7} \mathrm{erg} \mathrm{K}^{-1} \mathrm{~g}^{-1}$ is the universal gas constant, and plasma with $\gamma=5 / 3$ is assumed to constitute the flow. Three components of the flow velocity $v_{R}, v_{z}$, and $v_{\phi}$ are calculated in cylindrical coordinates $z, R$, assuming azimuthal symmetry, $\partial_{\phi}=0$.

Frequency-independent moments $E, \mathbf{F}$, which appear in the above set of radiation-hydrodynamic equations, are obtained by calculating angular moments from the frequency-integrated specific intensity, $I(\mathbf{r}, \Omega, v, t)$, and are given in Appendix A. Forces that are explicitly taken into account include radiation, grad pressure, and gas pressure, $\rho^{-1} \nabla p$.

These equations should be supplemented by the radiation force, $\mathbf{g}_{\mathrm{rad}}$, which is calculated from the following relation:

$$
\mathbf{g}_{\mathrm{rad}}=\frac{1}{c} \frac{\chi_{\mathrm{F}} \mathbf{F}}{\rho},
$$

where $\chi_{\mathrm{F}}=\chi_{\mathrm{a}}+\chi_{\mathrm{T}}$ is the total flux mean opacity consisting of absorption opacity, $\chi_{\mathrm{a}}$, and the Thomson scattering opacity, $\chi_{\mathrm{T}}$. As in Papers I and II, we did not differentiate between $\chi_{\mathrm{F}}, \chi_{\mathrm{P}}$, and $\chi_{\mathrm{E}}$. Radiation pressure on dust is most important from the dynamical point of view (Paper I). Note that in the infrared, the Rosseland mean opacity, $\kappa_{\mathrm{d}}=\chi_{\mathrm{d}} / \rho$ of dust with the temperature $10^{2}-10^{3} \mathrm{~K}$, is approximately 10-30 times larger than that of the electron Thomson opacities (Semenov et al. 2003). In this paper, when calculating radiation pressure force in Equation (5), we take into account only IR pressure on dust. Including UV force (in lines and on dust) is left for future studies. We adopt a simple procedure for the calculation of $\mathbf{g}_{\mathrm{rad}}$ : if the temperature of the dust $T_{\mathrm{d}}>T_{\text {sub }}$, where $T_{\text {sub }}$ is the dust sublimation temperature (see further in the text), the force is assumed to be zero.

Our description takes into account two types of radiation: infrared and X-ray. The transfer of X-rays is treated in a single-stream approximation. This implies that $\mathrm{X}$-rays are simply traced from the point source (i.e., corona) adopting $e^{-\tau_{X}}$ attenuation, where $\tau_{X}$ is an optical depth with respect to X-ray absorption (Dorodnitsyn et al. 2008). The distribution of the IR is calculated in a fluxlimited diffusion approximation. A detailed discussion about the validity of Equations (1)-(4) is given in Paper II. We briefly note that the closure relation between $\mathbf{F}$ and $E$ is obtained adopting the diffusion approximation:

$$
\mathbf{F}=-D \nabla E
$$

where if optical depth is large, $\tau \gg 1$, the diffusion coefficient reads

$$
D=c \lambda,
$$

where $\lambda=1 /(\kappa \rho)$ is the photon mean free path and $\kappa=\chi / \rho$. From Equations (5)-(7), one can see that in the diffusion regime the radiation force does not explicitly depend on the opacity.

The diffusion approximation tacitly assumes that optical depth $\tau \gtrsim 1$. Most of the torus where IR pressure is important indeed has $\tau_{\mathrm{d}}>1$, where $\tau_{\mathrm{d}}$ is the optical depth of the gas-dust mixture in the infrared.

If $\tau<1$, the diffusion approximation should be modified so a correct limiting behavior at $\tau<1$ is regained. One can see that without such modification, when $\tau \ll 1$, the mean free path $\lambda \rightarrow \infty, D \rightarrow \infty$, and $|\mathbf{F}| \rightarrow \infty$, which is in contradiction with a free-streaming limit, where it should be $|\mathbf{F}| \rightarrow c E$ (7). That is, when optical depth becomes small, or when $\rho \rightarrow 0$, the standard diffusion approximation is no longer applicable.

In order to correctly describe regions of small $\tau$, the standard approach is to adopt the flux-limited diffusion approximation (Alme \& Wilson 1974; Minerbo 1978; Levermore \& Pomraning 1981). In this approximation, $\lambda$ is replaced by $\lambda^{*}=\lambda \Lambda$, where $\Lambda$ is the flux limiter. The flux limiter that we adopted in Papers I and II and in the current work is that of Levermore \& Pomraning (1981):

$$
\Lambda=\frac{2+R_{\mathrm{LP}}}{6+3 R_{\mathrm{LP}}+R_{\mathrm{LP}}^{2}},
$$

where $R_{\mathrm{LP}}=\lambda|\nabla E| / E$. One can see that if $\tau \rightarrow 0$, then $R_{\mathrm{LP}} \rightarrow \infty$ and $|F| \sim c E$; if $\tau \gg 1, R_{\mathrm{LP}} \rightarrow 0$ and $\Lambda \rightarrow 1 / 3$.

\section{INTERACTION OF RADIATION AND THE WIND}

\subsection{Photoionization Equilibrium}

The premise of the current work is that the deposition of energy at parsec scale is dominated by external sources, i.e., by $\mathrm{UV}$ and X-rays from the central $\mathrm{BH}$ and inner accretion flow. Due to the high opacity of the dust-gas mixture, a significant part of the UV and soft X-rays is absorbed and reprocessed into infrared in a thin layer of thickness, $\delta l / R_{1 \mathrm{pc}} \simeq 1.3 \times 10^{-3} n_{7}^{-1}$ (Paper I). 
The dynamical time within the flow is usually much larger than the characteristic time of the photoionization and recombination. Thus, the ionization balance is determined by the condition of photoionization equilibrium. The condition of a photoionization equilibrium in the wind implies that the state of the gas can be reasonably parameterized in terms of one "ionization parameter," $\xi$ - the ratio of radiation energy density to baryon density (Tarter et al. 1969). In a popular form, it reads

$$
\xi=4 \pi F_{\mathrm{x}} / n \simeq 4 \times 10^{2} \cdot f_{\mathrm{x}} \Gamma M_{6} /\left(N_{23} r_{\mathrm{pc}}\right),
$$

where $F_{\mathrm{X}}$ is the local $\mathrm{X}$-ray flux, $L_{\mathrm{x}}$ is the $\mathrm{X}$-ray luminosity of the nucleus, $n$ is the gas number density, $N_{23}$ is the column density in $10^{23} \mathrm{~cm}^{-2}, f_{\mathrm{x}}$ is a fraction of the total accretion luminosity $L_{\mathrm{BH}}$ available in X-rays, and $\Gamma$ is a fraction of the total Eddington luminosity $L_{\text {Edd }}=1.25 \times 10^{44} M_{6}$, where $M_{6}$ is the mass of a $\mathrm{BH}$ in $10^{6} \mathrm{M}_{\odot}$. We adopt a simple attenuation model in which the local deposition of energy is proportional to $F_{\mathrm{x}}=L_{\mathrm{x}} e^{-\tau_{\mathrm{x}}} /\left(4 \pi r^{2}\right)$, where the optical depth at soft X-rays is integrated over a straight line connecting the point-source corona with a given parcel of gas $\tau_{\mathrm{x}}=\int \kappa_{\mathrm{x}} \rho d l$, and $\kappa_{\mathrm{x}}$ is the $\mathrm{X}$-ray opacity.

\subsection{Hot and Cold Gas}

To calculate photoionization balance, we take into account Compton and photoionization heating and Compton, radiative recombination, bremsstrahlung, and line cooling. The rates of these processes are calculated making use of the XSTAR photoionization code (Kallman \& Bautista 2001) for the incident spectrum, which is a power law with energy index $\alpha$. As the direct incorporation of the XSTAR subroutines into the hydrodynamics code is not feasible, the key ingredient of our method is to make use of approximate analytic formulae for the heating and cooling rates. These formulae approximate extensive tables of heating-cooling rates obtained from XSTAR; these approximations are similar to those of Blondin (1994), although modified by Dorodnitsyn et al. (2008) to incorporate better atomic data and power-law ionizing continuum with $\alpha=1$ :

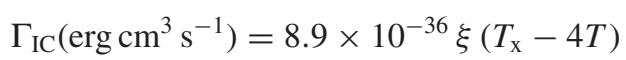

for the Compton heating-cooling,

$$
\Gamma_{\mathrm{x}}\left(\operatorname{erg~cm}^{3} \mathrm{~s}^{-1}\right)=1.5 \times 10^{-21} \xi^{1 / 4} T^{-1 / 2}\left(T_{\mathrm{x}}-T\right) T_{\mathrm{x}}^{-1}
$$

for the photoionization heating-recombination cooling, and

$$
\begin{aligned}
\Lambda\left(\mathrm{erg} \mathrm{cm}^{3} \mathrm{~s}^{-1}\right)= & 3.3 \times 10^{-27} T^{1 / 2} \\
& +\left(4.6 \times 10^{-17} \exp \left(-1.3 \times 10^{5} / T\right)\right. \\
& \left.\times \xi^{(-0.8-0.98 \alpha)} T-1 / 2+10^{-24}\right) \delta
\end{aligned}
$$

for the bremsstrahlung and line cooling. Formulae (10)-(12) were found to be in reasonable $(25 \%)$ agreement with numerical results.

A variety of physical and chemical processes influence the transformation of X-rays into IR if dense dusty, molecular gas is illuminated by external X-rays (Maloney et al. 1996; Krolik \& Lepp 1989). Rather than attempt to incorporate all these processes in our radiation-hydrodynamic framework, in the current paper we adopt several approximations that reproduce the qualitative behavior of low-temperature gas exposed to external X-ray radiation. At column densities $N$ smaller than $10^{24} \mathrm{~cm}^{-2}$, photons with energies below $10 \mathrm{keV}$ interact with the gas through photoelectric absorption, while Compton scattering dominates at higher energies. The total ionization per particle at a given point in the wind is proportional to $\xi_{\text {eff }}$, the ionization parameter that takes into account the frequencydependent attenuation of X-ray flux. Maloney et al. (1996) showed that heating and cooling rates of such X-ray-illuminated molecular gas depend on the modified ionization parameter, $\xi_{\text {eff }}=\xi / N_{22}^{0.9}=1.26 \times 10^{-1} F_{\mathrm{x}} /\left(n_{8} N_{22}^{0.9}\right)$, and several regimes are approximately distinguished: (1) the regime of highly ionized gas $\xi_{\text {eff }} \gg 1$. In this regime we make use of approximations for the heating and cooling rates (Equations (10)-(12)); (2) if $10^{-3} \ll \xi_{\text {eff }} \lesssim 1$, the gas is primarily atomic, and we also adopt heating-cooling rates (Equations (10)-(12)); (3) if the ionization parameter is small $\left(\xi_{\text {eff }} \ll \xi_{\mathrm{m}}=10^{-3}\right)$, the gas is largely molecular. This is where the most complexities due to chemistry and radiative interaction with the dust occur. The proper incorporation of this regime calls for consideration of all the radiative, chemical, and dust network of processes. This is challenging even without coupling to hydrodynamics.

In the regime when $\xi_{\text {eff }}<\xi_{\mathrm{m}}$, we assume that the molecular gas is in radiative equilibrium with dust, i.e., $T_{\mathrm{g}}=T_{\mathrm{d}}$, where equilibrium dust temperature is found from the attenuated X-ray flux, $F_{\mathrm{x}}$. Thus, we approximately take into account that dust can directly reprocess X-rays to IR contributing to the IR energy density, $E$. We approximately take this contribution into account, using equation (see Appendix B)

$$
\frac{d T_{\mathrm{r}}}{d t}=n_{\mathrm{d}} c \sigma_{d}\left(T_{\mathrm{d}}-T_{\mathrm{r}}\right) .
$$

Dust temperature is found from the approximate relation $T_{\mathrm{d}}=$ $\left(4 F_{\mathrm{X}} /(a c)\right)^{1 / 4}$ (note that $E=a T_{\mathrm{r}}^{4}$ ), where $F_{\mathrm{X}}$ is the local attenuated $\mathrm{X}$-ray flux. If the dust temperature $T_{\mathrm{d}}>T_{\text {sub }}$, where $T_{\text {sub }}$ is the dust sublimation temperature, then it is assumed that dust is destroyed and no conversion to IR occurs.

In our approach, the main difficulty in calculating the energy density of IR from X-rays results from a single fluid approximation. By having only one species (gas) and not evolving a separate dust component, we can mimic the interaction between dust and X-rays and calculate $T_{\mathrm{d}}$ by means of Equation (13), while additionally assuming the relation between $T_{\mathrm{d}}$ and $T$ in a dusty-molecular phase, in which, for simplicity, we assume $T=T_{r}$, with the latter calculated from Equation (13). Other dust characteristics in Equation (13) are as follows: $n_{\mathrm{d}}=\rho f_{\mathrm{d}} / m_{\mathrm{d}}$ is the dust grain number density, where $f_{\mathrm{d}}$ is the dust-to-gas mass ratio and $m_{\mathrm{d}}$ is the dust grain mass, and $\sigma_{\mathrm{d}}=\pi r_{\mathrm{d}}^{2}$ is the dust cross section, where $r_{\mathrm{d}}$ is the dust grain radius. Throughout our calculations, we adopt the following fiducial values for the parameters of dust: typical dust grain radius $r_{\mathrm{d}}=1 \times 10^{-4} \mathrm{~cm}$, the density of the dust grain $\rho_{\mathrm{d}}=1 \mathrm{~g} \mathrm{~cm}^{-3}, f_{\mathrm{d}}=1 \times 10^{-2}$, and above the sublimation temperature $T_{\text {sub }}=1500 \mathrm{~K}$ the dust is assumed to be destroyed. Equation (13) for $T_{\mathrm{d}}\left(F_{\mathrm{X}}\right)$ approximately describes how X-rays are converted into IR through reprocessing by dust.

If $\xi_{\text {eff }} \geqslant \xi_{\mathrm{m}}$ and $T<T_{\text {sub }}$, we assume that the exchange between $T_{\mathrm{r}}(E)$ and $T(e)$ is described by the balance between $D_{t}(E / \rho)$ and the last two terms in Equation (4). The gas temperature $T$ can contribute to IR and vice versa. For example, gas can contribute to the X-ray-IR conversion through cooling. Cooling can be due to radiative processes, i.e., the low energy limit of Equations (11) and (12), and due to "adiabatic" cooling due to fluid motion. 
The update of $E$ from Equation (13) is performed adopting operator splitting in the source step, i.e., before the update of Equation (4) is done. During the update over $d t$, the new $E_{\mathrm{r}}(t+d t)$ is calculated from Equation (13).

\section{METHODS}

To solve the system of Equations (1)-(4), we expanded the radiation-hydrodynamic framework described in our previous papers. The additions include the interaction of X-rays with dust grains and with gas. The coupling between the X-ray heating and cooling with hydrodynamics has been implemented and tested in our earlier works (Dorodnitsyn et al. 2008). Here, we have added physics that includes the direct interaction of X-rays with dust grains.

The radiation-hydrodynamic equations are solved using the ZEUS code (Paper II) with extensions taken from Dorodnitsyn et al. (2008). The radiation-hydrodynamic part is designed and tested in Papers I and Paper II and conforms with methods and structure of the original ZEUS code (Stone \& Norman 1992).

In the following, we briefly review the organizational structure of the code. Similar to the way pure hydrodynamics is treated in ZEUS, the radiation part is also split into the source and transport steps. Artificial viscosity is adopted to resolve discontinuities in the flow. In the source step the difference equations for $\partial E / \partial t$ and $\partial e / \partial t$ are solved, and in the transport step the advection of $E$ and $e$ is calculated. The update of $E$ and $e$ is calculated adopting a fully implicit scheme (see Paper II); this includes the solution of the diffusion problem and the $e \leftrightarrow E$ update, i.e., the update of gas and radiation energy densities due to radiation-gas interaction.

Similarly, in the X-ray-matter interaction step, heating $\gamma_{\mathrm{x}} \rightarrow e$ and cooling $e \rightarrow \gamma_{\mathrm{x}}$ due to Compton, radiative recombination, bremsstrahlung, and line cooling are also done fully implicitly in a separate update.

We make use of the $R, z$ cylindrical grid, which extends from $R_{\text {in }}$ to $R_{\text {out }}$ in the radial and from $z_{\text {in }}=0$ to $z_{\text {out }}=R_{\text {out }}$ in the vertical direction. The lower boundary at $z_{\text {in }}=0$ is intended to represent a thin accretion disk, which serves as an infinite reservoir of gas and dust. Wind can escape from all other boundaries, in principle, though the centrifugal barrier effectively prevents escape from the inner boundary. A fixed grid of size $200 \times 200$ is adopted for all calculations. Hydrodynamic BCs are as follows: at the left (innermost), right, and upper boundaries we adopt outflowing BCs.

At the equator, we fix the inflow speed $v_{0, j}$ at a small fraction of the speed of sound, leaving the density to adjust according to the continuity equation. Hereafter, index $j$ varies over $R$ and index $i$ over $z$. We allow $\rho_{i s, j}$ to vary and fix $v_{i s, j}$, that is, $\rho_{i s, j}=v_{i s+1, j} \rho_{i s+1, j} / v_{i s}$ and $v_{i s}=$ const, provided that $v_{i s} \ll v_{i s+1, \mathrm{~s}}$, where $v_{i s+1, \mathrm{~s}}$ is the sound speed; everywhere in the simulations we assumed $v_{i s} / v_{i s+1, \mathrm{~s}}=0.01$. Note that this is different from the implementation of the equatorial BC in Paper II, where it was assumed that $\rho_{i s, j} \sim R_{j}^{-p}$ and velocity was found from the continuity equation and only checked after the fact that $v_{i s, j} \ll v_{i s, \mathrm{~s}}$. The present implementation of BCs allows the disk to be flexible choosing mass loading of the wind. The initial distribution of density at the equator was taken to be $\rho_{0} r_{i s}^{-1}$, where $\rho_{0}=m_{p} n_{0}$ is the characteristic density and $m_{p}$ is proton mass. We find that numerical solution no longer resembles the initial equatorial distribution of $\rho$, and that solutions are not sensitive to the choice of $p$. Theoretically, since $\rho_{i s, j}$ is allowed to change, the final solution should only weakly depend on initial equatorial distribution of $\rho$. Practically, however, this is true only for those parts of the disk where the wind is developed. We also find that the present implementation of BCs produces a more stable solution near the equator, thus somewhat relaxing the restriction on the hydrodynamical time step.

The radiative $\mathrm{BCs}$ are free-streaming $F \sim c E$ at all boundaries except at the equator, where zero flux BCs are implemented.

\section{RESULTS}

\subsection{Dynamics of the Wind}

Deposition of radiation energy into the flow is controlled by the parameter $\eta=L_{\text {bol }} / L_{\text {Edd }}$. We calculate models for $\eta=\{0.05,0.1,0.3,0.4,0.5,0.6\}$. The innermost boundary of the computational domain is located at radius $R_{0}=0.5 \mathrm{pc}$. Other parameters are $M_{\mathrm{BH}}=1 \times 10^{7} M_{\odot}, f_{\mathrm{X}}=0.5$, and the initial scale density $n_{0}=1 \times 10^{8} \mathrm{~cm}^{-3}$. The evolution of the radiative flow was followed for 10-30 dynamical times, $t_{0} \simeq 1.5 \times 10^{11} r_{\mathrm{pc}}^{3 / 2} M_{7}^{-1 / 2}$ s.

In Papers I and II the temperature of the IR was prescribed at the boundary in a physically motivated way (to match the energetics of X-rays, $\left.f_{\mathrm{x}} L_{\mathrm{bol}}\right)$. The results obtained in Paper II are generally in good agreement with the present modeling. However, explicitly including X-rays in calculations reveals several important features of the flow that were absent in previous studies.

One of the results of the present study is that the pressure of the hot photoionized component is very important in shaping the IR flow. Excitation of such a hot, photoionized wind automatically mirrors the creation of the IR flow. The interface between the two components of the flow is seen in all simulations presented in this paper. In all cases, it takes the form of oblique shocks or contact discontinuities. A supersonically moving hot gas obliquely hits the slowly moving IR flow, creating a discontinuity (in most cases an oblique shock wave).

$$
\text { 5.1.1. Model with } L=0.6 L_{\mathrm{Edd}}
$$

Figure 1 shows the development of the radiation-driven disk wind for $\Gamma=0.6$. The outflow develops quickly after about one dynamical time, $t_{0} \simeq 4 \times 10^{4} \mathrm{yr}$. The mass-loss rate is $\langle\dot{M}\rangle \simeq$ $0.1-0.2 M_{\odot} \mathrm{yr}^{-1}$ on average and peaks at $\langle\dot{M}\rangle \simeq 1 M_{\odot} \mathrm{yr}^{-1}$ in the period of time between $4 \times 10^{3} \mathrm{yr}$ and $5 \times 10^{3} \mathrm{yr}$. The total computation spans from 0 to $8 \times 10^{3} \mathrm{yr}$. These averaged peak numbers include both failed wind and wind that has enough energy to escape to infinity.

The energetics of the wind is measured by computing the kinetic output of the wind at the outer boundary of the computational domain $\Sigma: v_{\text {kin }} \simeq 2 L_{\text {kin }} / \dot{M}$, where $L_{\text {kin }}=\int_{\Sigma} \rho v^{3} / 2 d \Sigma$ is the kinetic luminosity of the wind. For a radiation-driven wind we expect low values of $L_{\mathrm{kin}} / L_{\mathrm{bol}}$; here we have $L_{\mathrm{kin}} / L_{\mathrm{bol}} \simeq$ $5 \times 10^{-5}$ to $2 \times 10^{-4}$.

The averaged dynamics of the wind is described by the average bulk velocity of the flow $\langle v\rangle=\int_{V} \rho v d V / \int_{V} \rho d V$, where $V$ is the total or partial volume occupied by the flow. Here, the dense part of the flow has $\left\langle v_{z}\right\rangle \simeq$ a few $\mathrm{km} \mathrm{s}^{-1}$ and $\left\langle v_{R}\right\rangle \simeq$ a few $\times 10 \mathrm{~km} \mathrm{~s}^{-1}$. The maximum velocity reaches $1000 \mathrm{~km} \mathrm{~s}^{-1}$ in $R$ and over $600 \mathrm{~km} \mathrm{~s}^{-1}$ in the $z$-direction.

Figure 2 shows a color plot of the distribution of the gas temperature. The gas spans a vast range of temperatures from being in a cold molecular state to a photoionized, high-temperature 


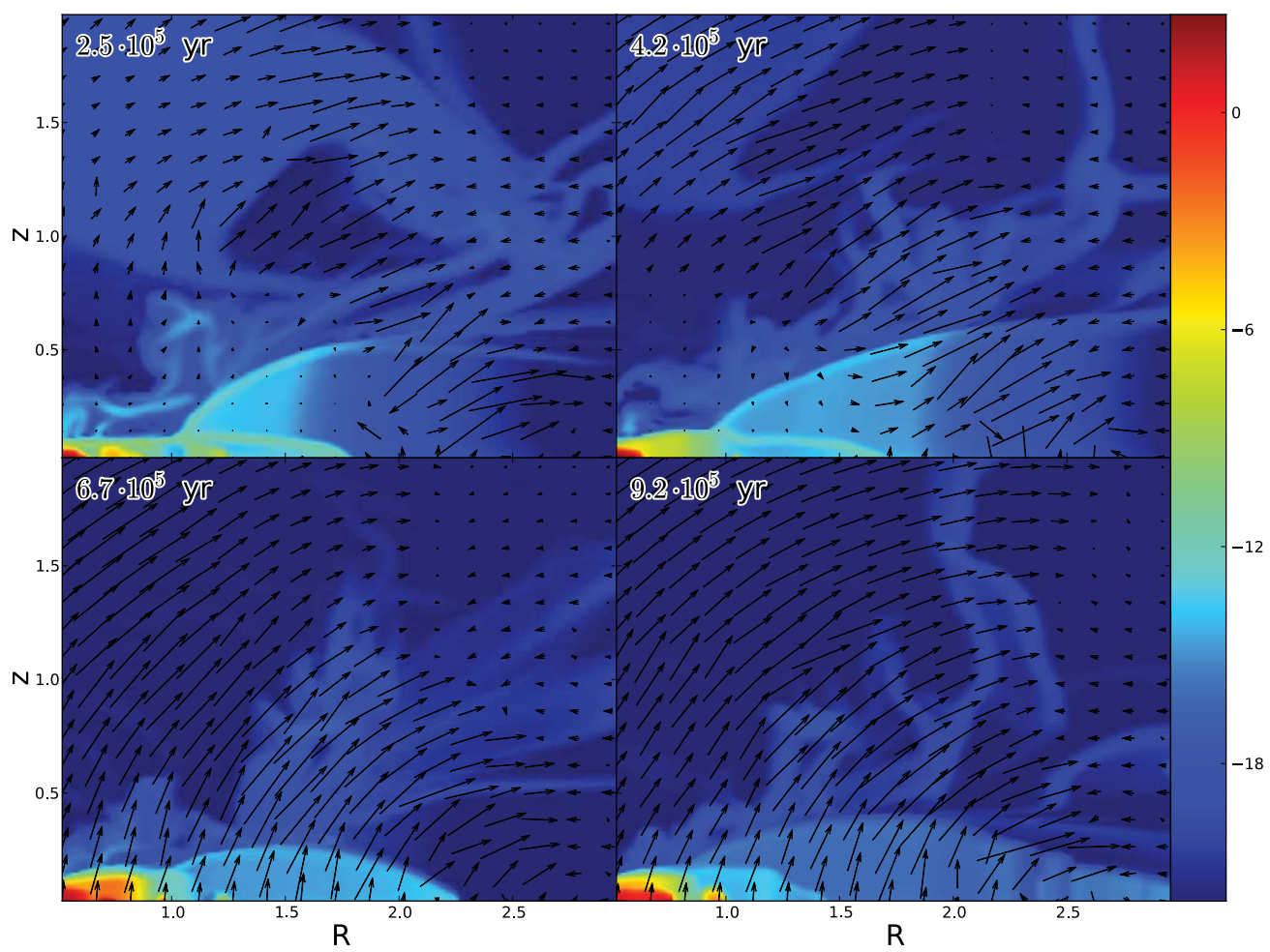

Figure 1. Color plot of the density, $\log \rho$, in $\mathrm{g} \mathrm{cm}^{-3}$ for the model with $L=0.6 L_{\mathrm{Edd}}$ shown at different times given in years. Axes: $z$ : distance from equatorial plane in parsecs; $R$ : distance from the $\mathrm{BH}$ in parsecs.

(A color version of this figure is available in the online journal.)

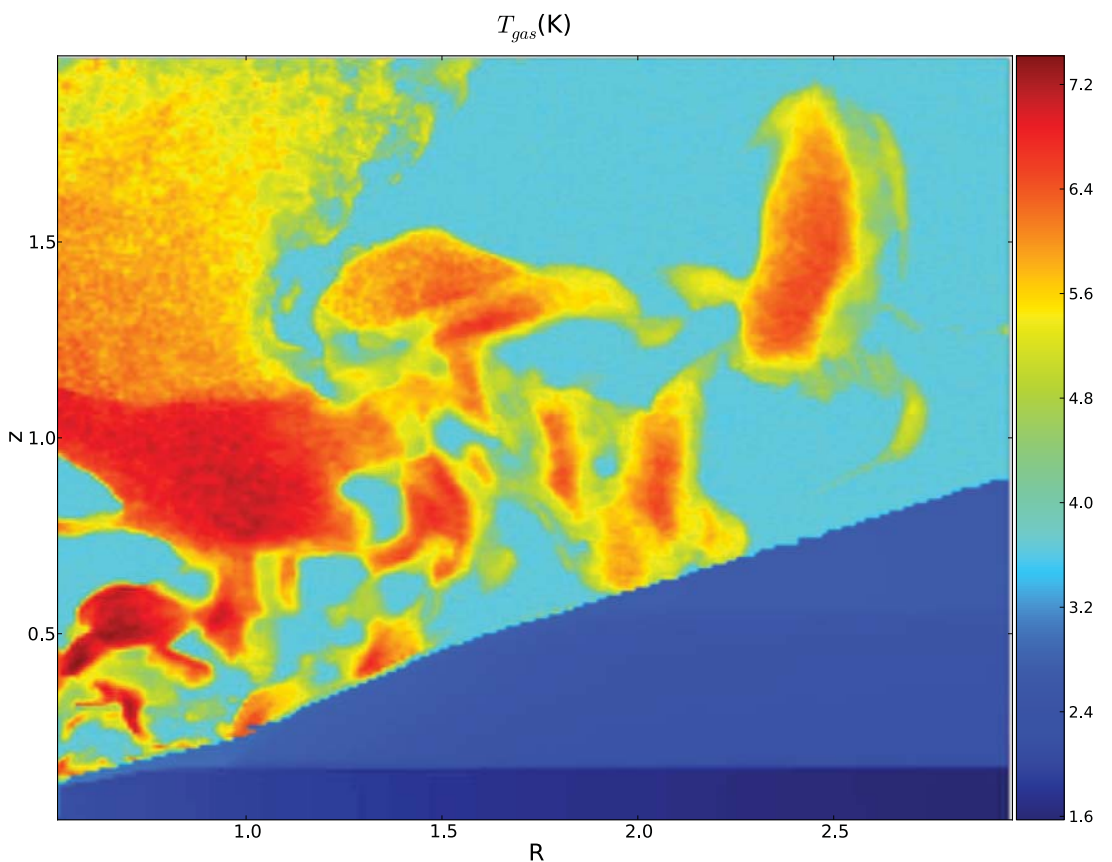

Figure 2. Color plot of the gas temperature, $\log T$, in $\mathrm{K}$ for the model with $L=0.6 L_{\mathrm{Edd}}$, after $4.2 \times 10^{5} \mathrm{yr}$. Axes: $z$ : distance from equatorial plane in parsecs; $R$ : distance from the $\mathrm{BH}$ in parsecs.

(A color version of this figure is available in the online journal.)

warm absorber flow. An apparent feature of our models is that the hot wind consists of large-scale inhomogeneities. The cold flow does not show such large-scale structure. Note that we have a very simple test if the gas is in the cold, molecular-dusty phase, $\xi_{\text {eff }}<\xi_{\mathrm{m}}$, and that if the hot component is not shown, the density structure is much more pronounced in the temperature plot.
It is of interest to address the question of whether there can be cold gas in a hot wind as well. However, the limited resolution of our studies does not allow us to provide a reliable answer to this question. We do not find the coexistence of hot and cold phases of gas in any appreciable quantities, but this does not exclude the possibility of such coexistence on length scales smaller than we can resolve. 


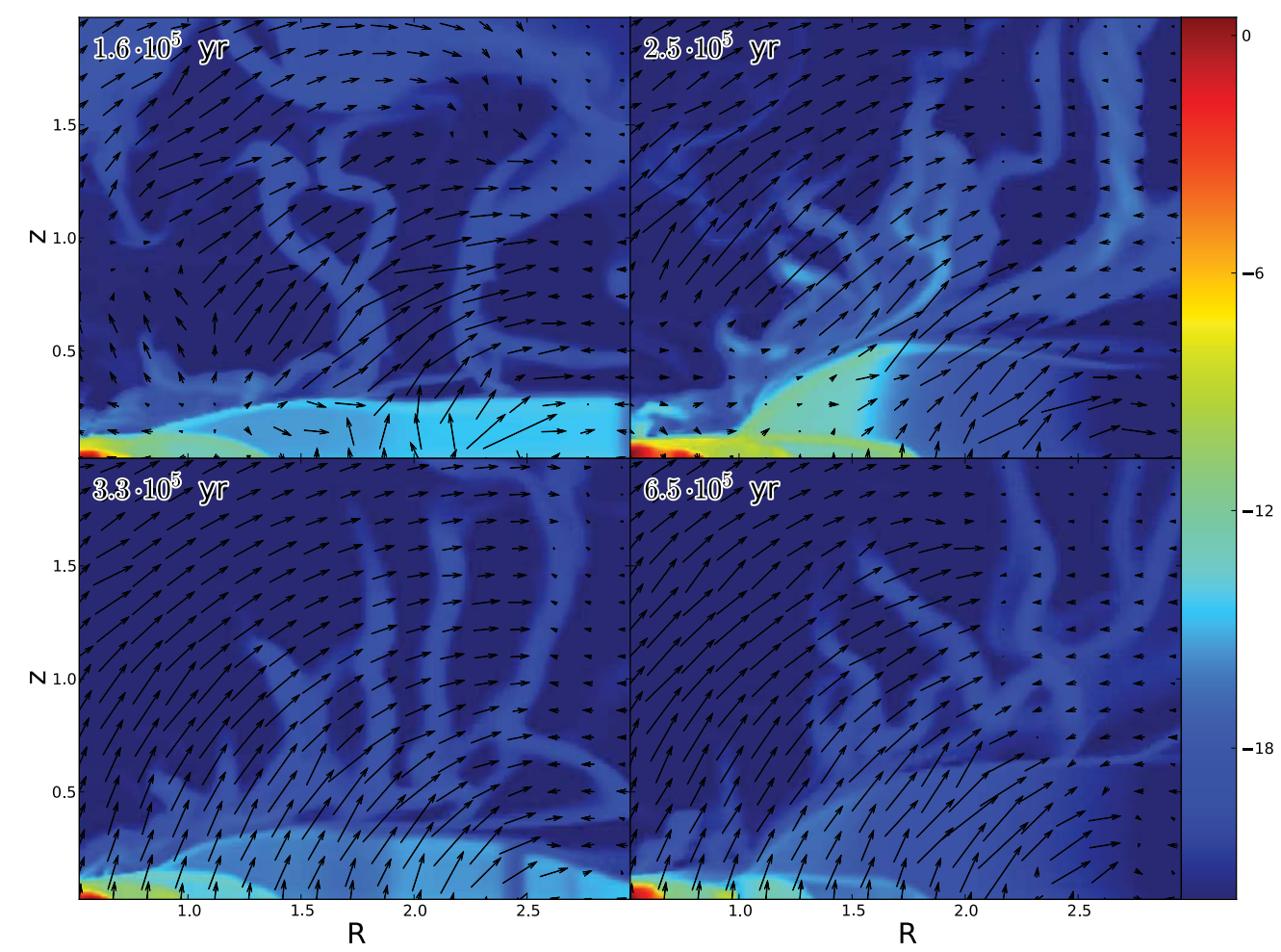

Figure 3. Color plot of the density, $\log \rho$, in $\mathrm{g} \mathrm{cm}^{-3}$ for the model with $L=0.5 L_{\mathrm{Edd}}$, shown at different times given in years. Axes: $z$ : distance from equatorial plane in parsecs; $R$ : distance from the $\mathrm{BH}$ in parsecs.

(A color version of this figure is available in the online journal.)

\subsubsection{Model with $L=0.5 L_{\mathrm{Edd}}$ : The Importance of the Hot Flow Pressure}

Figure 3, which shows a color plot of the density at different times, is very similar to Figure 1. One can distinguish approximately three regions in the flow: (1) a disk-like, geometrically thin and dense region extending to $R \sim 1.5 \mathrm{pc}$; (2) the IR-driven flow with lower density, separated by contact discontinuity from (3) a hot, photoionized flow. As $L$ decreases, so does the energetics of the wind. However, the vertical thickness of the dense IR-supported flow is determined by the delicate balance between (a) the $\rho v^{2}$ pressure of the hot component from above and (b) the pressure of IR-supported wind from below.

Decreasing $\Gamma$ from 0.6 to 0.5 causes the pressure of the hot flow to be less than in the solution shown in Figure 3. The balance between the pressure of the photoevaporated wind and IR-driven dusty flow is slightly shifted toward IR-dominated flow.

Figure 4 shows the two-dimensional distributions of the velocity components $v_{z}$ and $v_{R}$. The slowly outflowing wind is clearly seen; its well-defined boundary extends to $0.5 \mathrm{pc}$. The flow consists of a "core," i.e., slow-moving wind with radial velocities of a few $\times 10 \lesssim 100 \mathrm{~km} \mathrm{~s}^{-1}$, and $v_{z}$ much smaller, of the order of a few $1 \mathrm{~km} \mathrm{~s}^{-1}$. The fast lower-density component of the flow occupies most of the domain having velocities of the order of a few $\times 100 \lesssim 700 \mathrm{~km} \mathrm{~s}^{-1}$. The overall characteristics of the velocity field are similar to those of the model with $L=0.6 L_{\mathrm{Edd}}$. Not surprisingly, pumping less energy initially in $\mathrm{X}$-rays generates less powerful wind: $L_{\mathrm{kin}} / L_{\mathrm{bol}} \simeq 1 \times 10^{-5}$ to $7 \times 10^{-5}$.

The mass-loss rate $\langle\dot{M}\rangle \simeq 0.1 M_{\odot} \mathrm{yr}^{-1}$ with the peak $\langle\dot{M}\rangle \simeq 1.1 M_{\odot} \mathrm{yr}^{-1}$ in the period of time between $8 \times 10^{3} \mathrm{yr}$ and $10^{4} \mathrm{yr}$, and the computation spans from 0 to $8 \times 10^{3} \mathrm{yr}$.

\subsubsection{Model with $L=0.3 L_{\mathrm{Edd}}$}

Figure 5 demonstrates the evolution of the density for this model at different times. The disk-wind system goes through several stages: the domination of the hot wind, puffing up the IR-supported disk, squeezing of the latter toward the equator by the hot wind, building a high-density disk-like outflow, etc. As in previous examples, the system does not come to a quasistationary state, instead going through such episodes all over again.

An interesting feature that is seen at $t=2.5 \times 10^{5} \mathrm{yr}$ is in fact the residue from the contact discontinuity that existed at earlier times. The average velocity $\langle v\rangle \simeq$ a few $\times 172 \mathrm{~km} \mathrm{~s}^{-1}$. This is about $60 \%$ of the escape velocity, $U_{\text {esc }}$; the numbers are given for $t=20 t_{\mathrm{dyn}}$. The maximum velocity of the dusty flow is $242 \mathrm{~km} \mathrm{~s}^{-1}$.

The value of the mass-loss rate is remarkably similar to models with higher $\Gamma:\langle\dot{M}\rangle \simeq 0.1-0.2 M_{\odot} \mathrm{yr}^{-1}$ reaching $1 M_{\odot} \mathrm{yr}^{-1}$.

\subsubsection{Models with $L \lesssim 0.1 L_{\text {Edd }}$ Slow Accretion with Episodic Outflow}

When the radiation input falls below an approximate threshold value, no rigorous outflow is observed in our simulations. Instead, we see that episodic outbursts of the hot evaporative flow are excited from the inner parts of the disk. Here, we found this threshold luminosity to be $L \simeq 0.1 L_{\mathrm{Edd}}$. The results for the model for $L=0.1 L_{\text {Edd }}$ are shown in Figure 6 .

The density of this wind is too low to provide any considerable shielding from X-rays. Without much shielding, it is difficult to form a cold and dusty phase that can be accelerated by IR. Such an episodic outburst of the dense wind is followed by a gradual fallback of the dense component mostly in the $z$-direction toward the equatorial disk. 

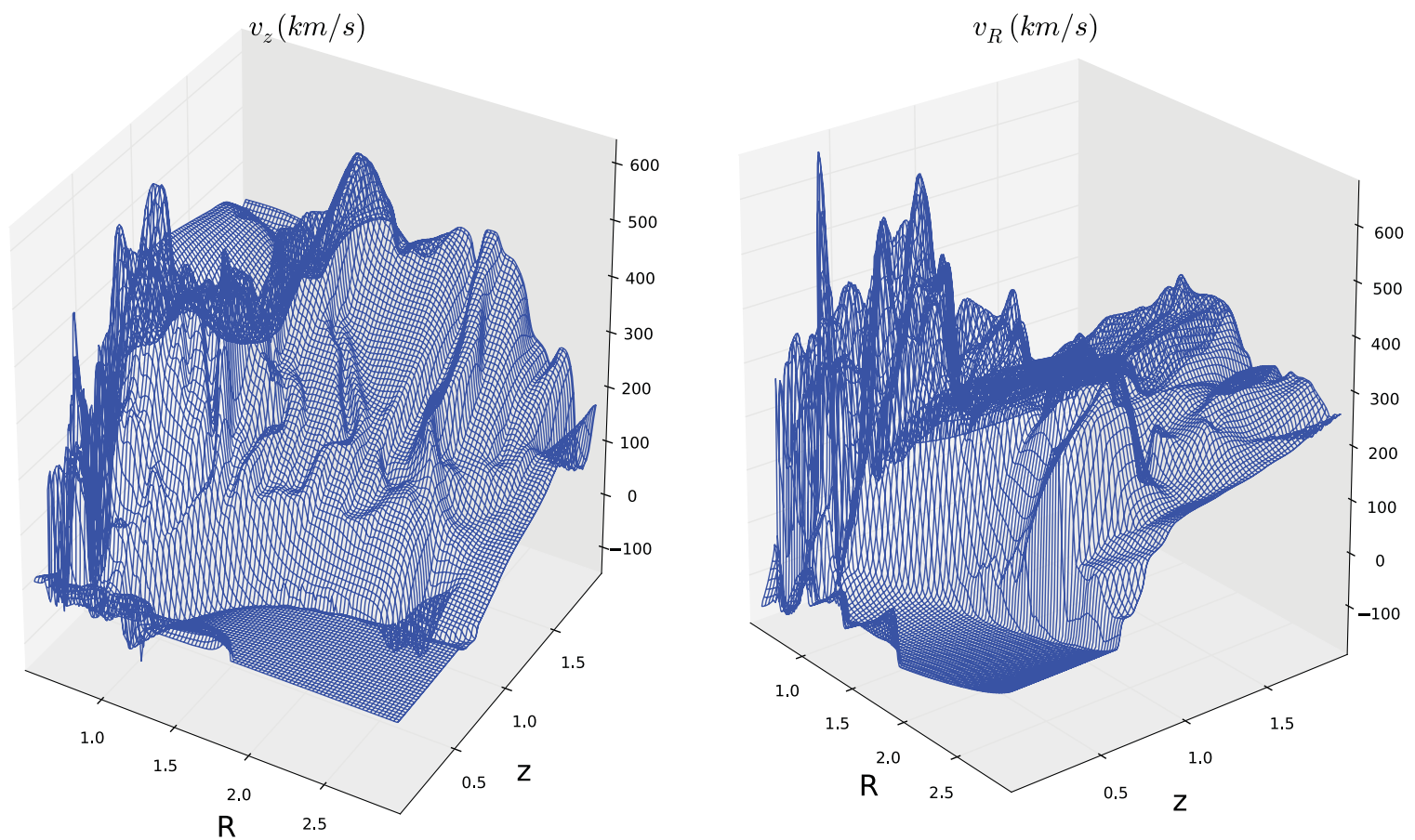

Figure 4. Surface plot of the $z$ - and $R$-velocity components. The model is shown with $L=0.5 L_{\mathrm{Edd}}$ and, $t=6.5 \times 10^{5} \mathrm{yr} ; R$ : distance from the $\mathrm{BH}$ in parsecs; $z$ : distance from the equatorial plane in parsecs.

(A color version of this figure is available in the online journal.)

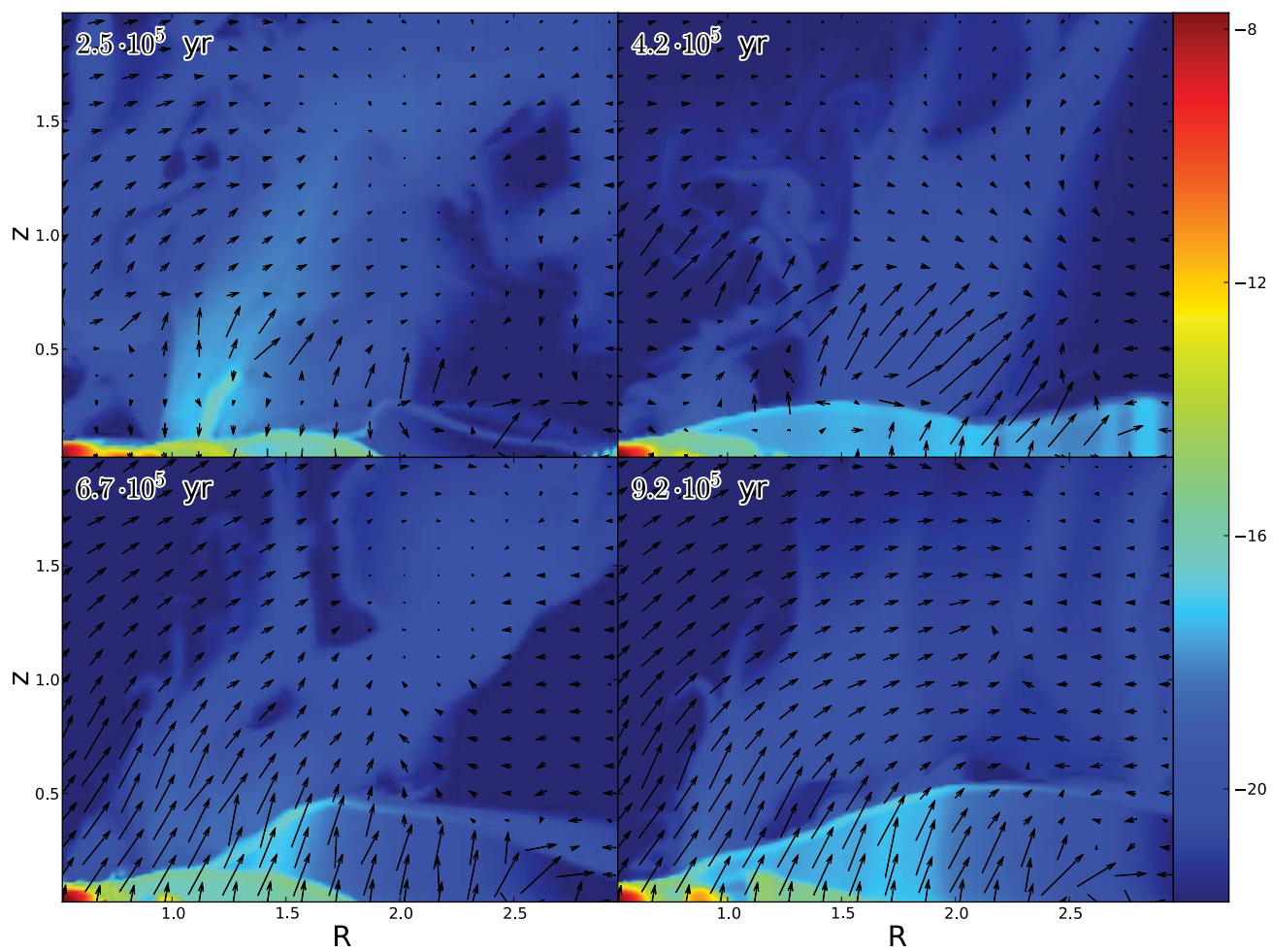

Figure 5. Color plot of the density, $\log \rho$, in $\mathrm{g} \mathrm{cm}^{-3}$ for the model with $L=0.3 L_{\text {Edd }}$ shown at different times given in years. Axes: $z$ : distance from equatorial plane in parsecs; $R$ : distance from the $\mathrm{BH}$ in parsecs.

(A color version of this figure is available in the online journal.)

The velocity of the hot wind, $v<U_{\text {esc }}$, skirts the denser flow and falls back toward the equator at larger radii, $R \simeq 2.5 \mathrm{pc}$. The density piles up there, and the shielding from $\mathrm{X}$-rays rapidly increases. Correspondingly, the ionization parameter drops below $\xi_{\mathrm{m}}$, and the cold IR-driven wind again has a chance to develop. Note that the vertical component of the radiation force is roughly $\mathbf{g}_{\text {eff, } z} \sim(d E / d r) \cos \theta$, where $r$ is the spherical radius and $\theta$ is the inclination angle, measured from the $z$-axis. Since the vertical extent of the IR wind is small, $\mathbf{g}_{\text {eff }, z}$ is small, and the dynamic pressure of the IR wind cannot overwhelm the 


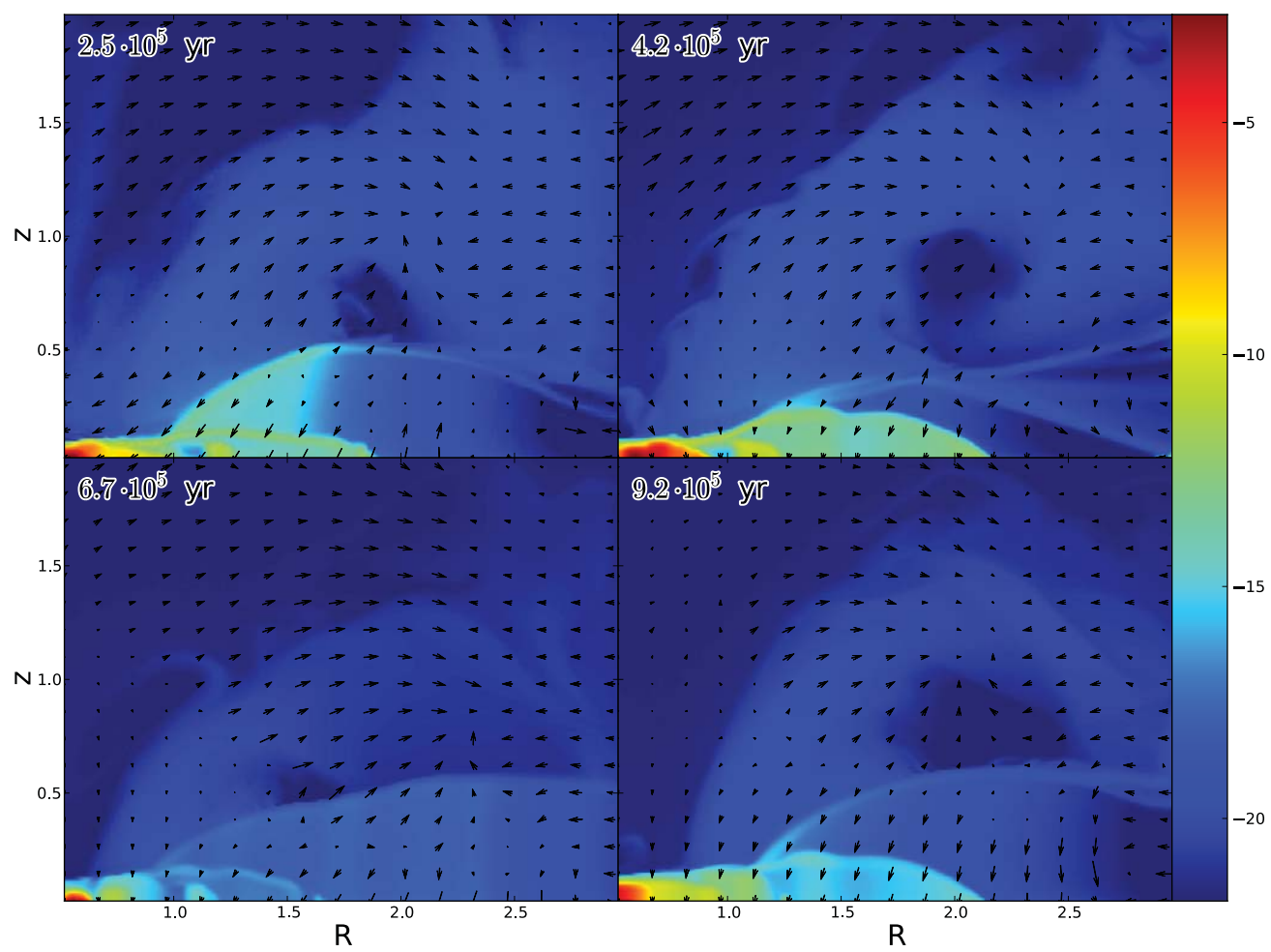

Figure 6. Color plot of the density, $\log \rho$, in $\mathrm{g} \mathrm{cm}^{-3}$ for the model with $L=0.1 L_{\mathrm{Edd}}$ shown at different times given in years. Axes: $z$ : distance from equatorial plane in parsecs; $R$ : distance from the $\mathrm{BH}$ in parsecs.

(A color version of this figure is available in the online journal.)

pressure of the hot component at higher $z$. The IR wind stalls and starts to slowly fall back (accrete) toward the equator. The density outside this dense IR-supported "pancake" drops, and the gas gets overionized again. The cold disk itself shrinks from both inside and outside.

We also calculated a model with $L=0.05 L_{\text {Edd }}$ and found that the general behavior of this model is similar to the previous one with $L=0.1 L_{\text {Edd }}$. The wind is episodic and intermittent with the "IR-supported pancake" phase.

It is difficult to access the dynamical properties of models with $L \lesssim 0.1 L_{\text {Edd }}$ because our equatorial BC excludes the influx of matter into the accretion disk. Strictly speaking, by assuming the thin accretion disk as a source of matter for the wind together with outflowing $\mathrm{BC}$ conditions, we assumed that the wind, even if very weak, is always present. We will further elaborate on this limitation in the following section.

\section{OBSCURING PROPERTIES}

We calculate Thomson optical depth $\tau_{\theta}=\int_{l} \kappa_{e} \rho d l$, where $l$ is measured along the line of sight at the angle $\theta$ from the vertical axis, and averaging is made over all calculated models for particular $\Gamma$. Processing different models, we are interested in an angle $\theta_{\mathrm{ph}}$ where $\tau_{\mathrm{ph}}=\tau\left(\theta_{\mathrm{ph}}\right)=1$, i.e., when the wind becomes opaque.

In the following we summarize results, listing models in the order from low to high luminosities. When $\Gamma=0.05$, the optical depth rises sharply from 0.1 to 0.5 at $\langle\theta\rangle \simeq 75^{\circ}$ (hereafter $\theta_{\text {edge }}$ ), and the photosphere is at $\left\langle\theta_{\mathrm{ph}}\right\rangle \simeq 78^{\circ}$.

Increasing the luminosity to $\Gamma=0.1$ causes the dense part of the wind to become thicker in vertical extent, optical depth rises at the angle $\theta_{\text {edge }} \simeq 73^{\circ}$, and the photosphere is at $\left\langle\theta_{\mathrm{ph}}\right\rangle \simeq 75^{\circ}$.
At $\Gamma=0.3$ optical depth rises sharply from 0.1 at $\langle\theta\rangle \simeq 73^{\circ}$, $\left\langle\theta_{\text {ph }}\right\rangle \simeq 75^{\circ}$, and $\theta_{\text {edge }} \simeq 73^{\circ}$.

For the model with $\Gamma=0.5$ the photosphere is at $\left\langle\theta_{\mathrm{ph}}\right\rangle \simeq 75^{\circ}$ and the torus edge is at $\theta_{\text {edge }} \simeq 73^{\circ}$.

Figure 7 shows a color plot of a radial Thomson optical depth measured from a $\mathrm{BH}$ to a given point of the computational domain. The model shown has $L=0.6 L_{\text {Edd }}$. This is the most luminous model we have calculated. It has the photosphere located at $\left\langle\theta_{\mathrm{ph}}\right\rangle \simeq 72^{\circ}$ and the torus edge at $\theta_{\text {edge }} \simeq 71^{\circ}$.

\section{MASS-LOSS RATE}

The opacity of dusty plasma in the infrared domain is 10-20 times that of electron scattering (Semenov et al. 2003). Such huge opacity makes it possible for the AGN to have a strong wind, radiating at a fraction of Eddington luminosity (defined with respect to Thomson scattering; see Paper I for an estimate of an outflow onset).

Mass-loss rate from the IR-driven portion of the wind can be estimated by combining the results from a theory of stellar winds and some of the approximations adopted in Paper I. From the stellar wind theory, the mass-loss rate from a spherically symmetric wind can be found from the following approximate relation (Lamers \& Cassinelli 1999):

$$
\dot{M} /(4 \pi) \simeq L_{\mathrm{IR}} / c\left(\Gamma_{\mathrm{IR}}-1\right) /\left(4 \pi v^{\infty} \Gamma_{\mathrm{IR}}\right) \tau_{\mathrm{w}},
$$

where $\Gamma_{\mathrm{IR}}=L_{\mathrm{X}} / L_{\mathrm{Edd}, \mathrm{IR}}, v^{\infty}$ is the wind terminal velocity, and $\tau_{\mathrm{w}}$ is the wind optical depth in IR.

For simplicity, as in Paper I, in this section we assume that all incident $\mathrm{UV}$ and $\mathrm{X}$-ray radiation is reprocessed within a narrow conversion layer in which effective temperature, $T_{\text {eff }}$, can be found from the following approximate relation: $\alpha \Gamma \eta_{\mathrm{X}} F_{\mathrm{Edd}}=$ $\sigma T_{\mathrm{eft}}^{4}$, where $\Gamma=L / L_{\mathrm{Edd}}, \eta_{\mathrm{X}}=0.5$ is the fraction of X-ray 


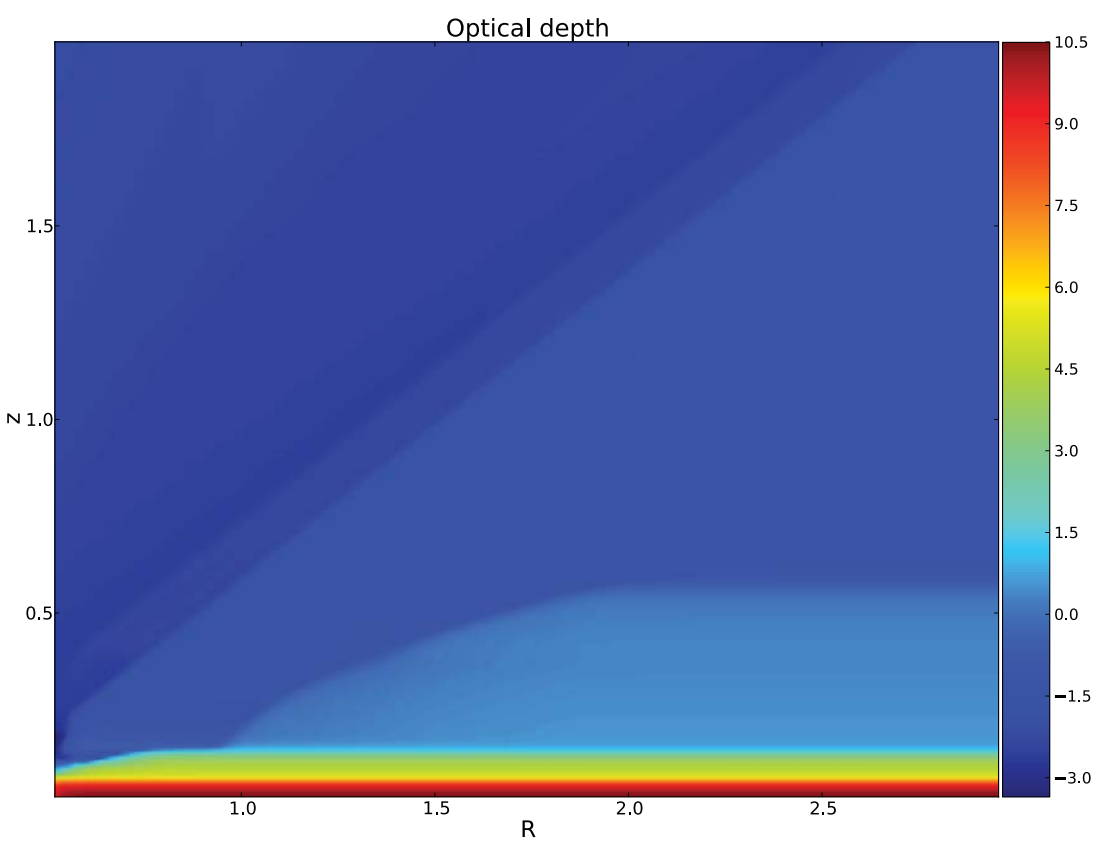

Figure 7. Color plot of the radial Thomson optical depth, $\log \tau$, from a BH toward a given point. Axes: $z$ : distance from equatorial plane in parsecs; $R$ : distance from the $\mathrm{BH}$ in parsecs.

(A color version of this figure is available in the online journal.)

radiation from the total radiation, and the fraction $\alpha \simeq 0.5$ of the incident flux is re-emitted outwardly. Adopting the above, one obtains $\Gamma_{\mathrm{IR}}=\alpha \Gamma \eta_{\mathrm{X}} \kappa_{\mathrm{d}} / \kappa_{\mathrm{e}}$, which is approximately 1.25 for $M_{\mathrm{BH}}=10^{7} M_{\odot}, L=0.5 L_{\mathrm{ddd}}$, and $\kappa_{\mathrm{d}}=10 \kappa_{\mathrm{e}}$. The value of $v^{\infty}$ is estimated while neglecting $P_{\mathrm{g}}$ in favor of the radiation pressure: $v^{\infty}=\sqrt{2 G M / r_{0}\left(\Gamma_{\mathrm{IR}}-1\right)} \simeq 207 \mathrm{~km} \mathrm{~s}^{-1}$ adopting the above set of parameters plus the wind launching radius $R_{0}=0.5 \mathrm{pc}$, and assuming that the wind occupies a wedge-like (in a quarter of the domain) space of an opening angle of $75^{\circ}$, we arrive at the estimate $M \simeq 3 \tau_{w} M_{\odot} \mathrm{yr}^{-1}$. Monte Carlo simulations show that the factor $\tau_{\mathrm{w}}$ can reach 1-5 for an AGN radiating at a fraction of $L_{\text {Edd }}$ (Roth et al. 2012), meaning the potential for the wind tori to reach a mass-loss rate of $10 M_{\odot} \mathrm{yr}^{-1}$. We do not see such mass-loss rates in our simulations, which may indicate that instead of being blown away, as it certainly would in a spherically symmetric case, the gas is effectively ablated toward the equatorial plane by the pressure of the hot gas component. As the latter is itself the result of interaction with radiation, one can say that radiation effectively clears its way through the opening of the torus.

\section{DISCUSSION}

The following average properties of our wind solutions can be summarized:

1. The total mass-loss rate in the IR-driven and warm absorber flows is between $\langle\dot{M}\rangle \simeq 0.1 M_{\odot} \mathrm{yr}^{-1}$ and $\langle\dot{M}\rangle \simeq$ $1.5 M_{\odot} \mathrm{yr}^{-1}$ depending on the time of the evolution of the wind. This also includes the failed wind. The amount of gas that has enough energy to escape to infinity is in good accord with the energetics derived from accretion. Note that approximately $1.6 \times 10^{-1}$ to $1.3 \times 10^{-2} M_{\odot} \mathrm{yr}^{-1}$ are required to be accreted through a geometrically thin accretion disk to support our models. There is also no problem of depletion of the torus since we have an infinite reservoir of matter in a razor-thin equatorial disk.
2. The IR-driven wind has a velocity in the range of a few $x$ $10-200 \mathrm{~km} \mathrm{~s}^{-1}$, and the fast hot wind has a velocity in the range of $400-800 \mathrm{~km} \mathrm{~s}^{-1}$.

3. The transmission properties are similar between different models. The characteristic angle at which the wind becomes opaque to Thomson scattering is about $72^{\circ}-75^{\circ}$ and is determined by the balance between the hot, photoionized wind and the cold wind supported by the IR pressure on dust. It is interesting that the geometry of the torus is close to one obtained in Dorodnitsyn et al. (2008) at late times when the pressure of the hot, evaporative component becomes important.

4. Both hot and cold components of the wind are non-stationary.

Models such as Konigl \& Kartje (1994), Elitzur \& Shlosman (2006), Emmering et al. (1992), Everett (2005), Keating et al. (2012), and Bottorff et al. (2000) are developed on the premise of (1) the existence of a strong global magnetic field and (2) using the prescription of self-similarity. To a certain extent this limits the value of the direct comparison of our results with theirs. Indirect comparison would include performing a detailed analysis of the spectral properties of the IR-driven "wind torus" and performing our simulations for the parameter range attributed to a well-studied source (such as NGC 5548, in the case of Bottorff et al. 2000). Those are the next things to do in order to assess the validity of the current model, and both of these goals are a natural continuation of the present studies. We will elaborate on observational properties and the implications of the time-dependent behavior of our solutions in a following paper of this series.

\section{CONCLUSIONS}

Our studies of AGN infrared-driven winds have proceeded incrementally. In Paper I, we considered a toy model that roughly approximated an infrared-driven obscuring wind in AGNs. An important prediction that resulted was that if the temperature of 
the dusty plasma in the rotating torus exceeds some characteristic value, the IR pressure on dust will inevitably overwhelm gravity, creating an outflow. However, this effective temperature, which approximately reads as $T_{\text {eff }}=(G M \rho / a r)^{1 / 4} \simeq$ $312\left(n_{5} M_{7} / r_{\mathrm{pc}}\right)^{1 / 4}-987\left(n_{7} M_{7} / r_{\mathrm{pc}}\right)^{1 / 4} \mathrm{~K}$, was derived analytically from a simplified model.

In Paper I, we combined calculations of stationary onedimensional motion in the $z$-direction with two-dimensional calculations of the radiation field. The latter was calculated adopting a two-dimensional flux-limited diffusion approximation. In Paper II, we relaxed the assumption of one-dimensional stationary motion and adopted a $2.5 \mathrm{D}$ time-dependent picture. One important "toy-model" simplification still remained, however; there was no X-ray radiation explicitly taken into account. Instead, we calculated the effective temperature of the gas at the boundary of the computational domain. In the present work, we relaxed this assumption and included X-rays in the global picture explicitly.

Despite these advances, our work still does not consider some potentially important physical processes.

The assumption of a razor-thin disk is idealized. An accretion disk at parsec scales is likely self-gravitating, possibly affected by gravitothermal instability (i.e., Gammie 2001). This may provide a local source of energy and maintain a Toomre parameter $\Omega_{\mathrm{T}} \sim 1$ (Toomre 1964). In a marginally gravitationally unstable thin disk, the non-axisymmetric perturbations lead to angular momentum transport that can be described by an effective viscosity (i.e., Paczynski 1978; Shlosman et al. 1989, 1990; Rafikov 2009). Self-consistent models of geometrically thick disks in AGNs at parsec scales call for the effects of selfgravity and radiation physics in three dimensions. This difficult endeavor can be the subject of future research. Another complication is that the problem of parsec-scale winds may be intrinsically connected with the problem of accretion at parsec scale. Relaxing the assumption of an equatorial thin disk and treating accretion adopting an effective $\alpha$ prescription is a possible next step in the development of the current model.

The assumption of a razor-thin disk as a source of matter underpins the current study. Certainly such a distribution of matter is in many respects most unfavorable for the formation of radiation-driven flow, owing to the geometry of X-ray illumination. Thus, we expect that if the wind does form for certain $\Gamma$ in the thin-disk case, then we may expect that it will also form in less idealized circumstances. To relax the assumption of a razor-thin disk, one would need to abandon the assumption of equatorial symmetry and to include an accretion disk in self-consistent consideration.

Starting from Paper I, we see that much of the massive wind does not leave the potential well of the $\mathrm{BH}$. This conclusion is confirmed through Paper II, as well as in the present work. The precise fate of this gas is beyond the scope of these works, but the results suggest the following: if the accreted gas is rejected by the $\mathrm{BH}$, at least some of it will return for additional attempts.

We conclude with a mix of results and ideas driven by our current studies:

1. The distribution of radiation force inside the dusty component of the flow depends on the shape of the photosphere where X-rays are converted into IR and can only be calculated in multi-dimensional simulations. This work includes both X-rays and IR in 2.5D time-dependent, hydrodynamic simulations.

2. We do not observe a rotationally supported quasi-static torus in our simulations, although our simulated winds are likely to resemble some of the observed properties of such tori.

3. The flow in the current simulations is more complex and time-dependent than that of our previous studies, where we did not include X-rays explicitly.

4. If geometrically thick dusty obscuration develops, then a hot photoionized flow with velocities of $100-700 \mathrm{~km} \mathrm{~s}^{-1}$ accompanies it. X-ray warm absorbers are the evaporative flow both originating from the disk and also evaporated from such a cold, dusty component.

5. We speculate that large-scale motions with $v_{z}= \pm($ a few $) \times$ $100 \mathrm{~km} \mathrm{~s}^{-1}$ originating from a spatially varying wind such as we have calculated may be seen in maser emission in nearby bright type II AGNs.

This research was supported by an appointment at the NASA Goddard Space Flight Center, administered by CRESST/UMD through a contract with NASA, and by grants from the NASA Astrophysics Theory Program 10-ATP10-0171. G.S. Bisnovatyi-Kogan acknowledges the support from the Russian Foundation for Basic Research (RFBR Grant 11-02-00602).

\section{APPENDIX A}

Frequency-integrated moments $E, \mathbf{F}$, which appear in the above set of Equations (1)-(4), are obtained by calculating angular moments from the frequency-integrated specific intensity, $I(\mathbf{r}, \Omega, v, t)$ :

$$
\begin{aligned}
& E(\mathbf{r}, \mathbf{t})=\frac{1}{c} \int_{0}^{\infty} d v \oint d \Omega I(\mathbf{r}, \Omega, v, t), \\
& \mathbf{F}(\mathbf{r}, \mathbf{t})=\int_{0}^{\infty} d v \oint d \Omega \hat{\mathbf{n}} I(\mathbf{r}, \Omega, v, t) .
\end{aligned}
$$

The frequency-independent radiation pressure tensor $\mathbf{P}$ is found from

$$
\mathbf{P}(\mathbf{r}, t)=\frac{1}{c} \int_{0}^{\infty} d v \oint d \Omega \hat{\mathbf{n}} \hat{\mathbf{n}} I(\mathbf{r}, \Omega, v, t) .
$$

\section{APPENDIX B}

Dust directly reprocesses X-rays to IR, and we approximately take this into account. The amount of energy absorbed by dust in a volume $d^{3} x$ is:

$$
\frac{1}{d^{3} x} \frac{d E_{\mathrm{x}}^{\nu}}{d t}=\alpha n_{\mathrm{d}} \int \sigma_{\mathrm{x}} I_{\nu \mathrm{x}} d \Omega d \nu \simeq \alpha n_{d} \sigma_{\mathrm{x}} c u_{x},
$$

where $u_{\mathrm{x}}$ is the energy density of X-rays, $\sigma_{\mathrm{x}}=\chi_{\mathrm{x}} / n_{\mathrm{d}}$, and the number density of dust grains reads $n_{d}=f_{\mathrm{d}} \rho / m_{\mathrm{d}}$, where $m_{\mathrm{d}}$ is the mass of a grain and $f_{\mathrm{d}}$ is the dust-to-gas mass ratio. We make the further simplifying assumption that the dust grain is being instantaneously heated to the effective temperature $T_{\mathrm{d}, \mathrm{x}}$.

The contribution from the dust to the IR energy density (or, equivalently, to the temperature $T_{\mathrm{r}}$ ) is calculated from the following equation:

$$
\frac{d T_{\mathrm{r}}}{d t}=n_{\mathrm{d}} c \sigma_{d}\left(T_{\mathrm{d}, \mathrm{x}}-T_{\mathrm{r}}\right) .
$$

Assuming that $T_{\mathrm{d}, \mathrm{x}}$ is constant over the time interval $[t, t+d t]$, the update for radiation is $T_{\mathrm{d}, \mathrm{x}}(t+d t)=T_{\mathrm{d}, \mathrm{x}}\left(1-e^{-d t / t_{d}}\right)+$ $-e^{-d t / t_{d}} T_{\mathrm{d}, \mathrm{x}}(t)$. Note that $E=a T_{\mathrm{r}}^{4}$. Equation (B) is used in an operator-split fashion to update $E$; prior to this all other terms in Equation (4) are updated. 


\section{REFERENCES}

Alme, M. L., \& Wilson, J. R. 1974, ApJ, 194, 147

Antonucci, R. R. J. 1984, ApJ, 278, 499

Antonucci, R. R. J., \& Miller, J. S. 1985, ApJ, 297, 621

Bannikova, E. Y., Vakulik, V. G., \& Sergeev, A. V. 2012, MNRAS, 424, 820

Beckert, T., \& Duschl, W. J. 2004, A\&A, 426, 445

Blondin, J. M. 1994, ApJ, 435, 756

Bottorff, M. C., Korista, K. T., \& Shlosman, I. 2000, ApJ, 537, 134

Czerny, B., \& Hryniewicz, K. 2011, A\&A, 525, L8

Dorodnitsyn, A., Bisnovatyi-Kogan, G. S., \& Kallman, T. 2011, ApJ, 741, 29

Dorodnitsyn, A., Kallman, T., \& Bisnovatyi-Kogan, G. S. 2012, ApJ, 747, 8

Dorodnitsyn, A., Kallman, T., \& Proga, D. 2008, ApJ, 687, 97

Elitzur, M. 2008, New Astron. Rev., 52, 274

Elitzur, M., \& Shlosman, I. 2006, ApJ, 648, L101

Emmering, R. T., Blandford, R. D., \& Shlosman, I. 1992, ApJ, 385, 460

Everett, J. E. 2005, ApJ, 631, 689

Gammie, C. F. 2001, ApJ, 590, 174

Jaffe, W., Meisenheimer, K., Röttgering, H. J. A., et al. 2004, Nature, 429, 47

Kallman, T. R., \& Bautista, M. A. 2001, ApJS, 133, 221

Keating, S. K., Everett, J. E., Gallagher, S. C., \& Deo, R. P. 2012, ApJ, 749,32

Konigl, A., \& Kartje, J. F. 1994, ApJ, 434, 446

Krolik, J. H. 2007, ApJ, 661, 52

Krolik, J. H., \& Begelman, M. C. 1988, ApJ, 329, 702

Krolik, J. H., \& Lepp, S. 1989, ApJ, 347, 179
Lamers, H. J. G. L. M., \& Cassinelli, J. P. 1999, Introduction to Stellar Winds (Cambridge: Cambridge Univ. Press)

Levermore, C. D., \& Pomraning, G. C. 1981, ApJ, 248, 32

Lovelace, R. V. E., Romanova, M. M., \& Biermann, P. L. 1998, A\&A, 338, 856

Maloney, P. R., Hollenbach, D. J., \& Tielens, A. G. G. M. 1996, ApJ, 466, 561

Mihalas, D., \& Mihalas, B. W. 1984, in Foundations of Radiation Hydrodynamics, ed. D. Mihalas \& B. W. Mihalas (New York: Oxford Univ. Press)

Minerbo, G. N. 1978, J. Quant. Spec. Radiat. Transf., 20, 541

Nenkova, M., Sirocky, M. M., Ivezić, Ž., \& Elitzur, M. 2008, ApJ, 685, 147

Paczynski, B. 1978, Acta Astron., 28, 91

Phinney, E. S. 1989, in NATO ASIC Proc. 290: Theory of Accretion Disks, ed. F. Meyer (Dordrecht: Kluwer), 457

Rafikov, R. R. 2009, ApJ, 704, 281

Richards, G. T., Lacy, M., Storrie-Lombardi, L. J., et al. 2006, ApJS, 166, 470

Roth, N., Kasen, D., Hopkins, P. F., \& Quataert, E. 2012, ApJ, 759, 36

Rowan-Robinson, M. 1977, ApJ, 213, 635

Sanders, D. B., Phinney, E. S., Neugebauer, G., Soifer, B. T., \& Matthews, K. 1989, ApJ, 347, 29

Semenov, D., Henning, T., Helling, C., Ilgner, M., \& Sedlmayr, E. 2003, A\&A, 410,611

Shlosman, I., Begelman, M. C., \& Frank, J. 1990, Nature, 345, 679

Shlosman, I., Frank, J., \& Begelman, M. C. 1989, Nature, 338, 45

Stone, J. M., \& Norman, M. L. 1992, ApJS, 80, 753

Tarter, C. B., Tucker, W. H., \& Salpeter, E. E. 1969, ApJ, 156, 943

Toomre, A. 1964, ApJ, 139, 1217

Tristram, K. R. W., Meisenheimer, K., Jaffe, W., et al. 2007, A\&A, 474, 837

Urry, C. M., \& Padovani, P. 1995, PASP, 107, 803 